Supporting Information

for

\title{
Direct Observation of the Preference of Hole Transfer over Electron Transfer for Radical Ion Pair Recombination in Donor-Bridge-Acceptor Molecules
}

Zachary E. X. Dance, Michael J. Ahrens, Amy M. Vega, Annie Butler, David W. McCamant, Mark A. Ratner*, and Michael R. Wasielewski*

Department of Chemistry, Argonne-Northwestern Solar Energy Research (ANSER) Center, and International Institute for Nanotechnology

Northwestern University, Evanston, Illinois 60208-3113

\section{Synthesis}

Proton nuclear magnetic resonance spectra were recorded on a Mercury 400 or Inova 500 NMR spectrometer using TMS as an internal standard. High resolution electrospray and fast atom bombardment mass spectra were obtained with the 70-SE-4F and Q-Tof Ultima mass spectrometers at the University of Illinois at Champaign-Urbana. Solvents and reagents were used as received except where otherwise noted. Flash and thin-layer chromatography was performed using Sorbent Technologies (Atlanta, GA) silica gel. All solvents were spectrophotometric grade. Toluene was purified by passing it through a series of $\mathrm{CuO}$ and alumina columns (GlassContour). Chloroform was washed three times with deionized water, dried over sodium sulfate, and then passed through a column of basic alumina.

The following compounds have been previously reported:

1. $N$-(n-octyl)-naphthalene-1,8-dicarboxyanhydride-4,5-dicarboximide: ${ }^{1}$

2. 4-amino-4'-bromobiphenyl ${ }^{2}$ 


\section{4-amino-4"'-bromoterphenyl ${ }^{2}$}

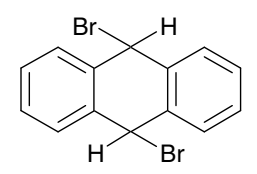

9,10-dibromo-9,10-dihydroanthracene $(\mathbf{A}) .^{3}$ Anthracene $(5.012 \mathrm{~g}, 28.12 \mathrm{mmol})$ was placed in a flame-dried $250 \mathrm{ml}$ round bottom flask with $55 \mathrm{ml}$ dry carbon disulfide. The reaction flask was cooled to $0^{\circ} \mathrm{C}$ with a water / ice bath under a nitrogen atmosphere. Bromine $(1.3 \mathrm{ml}, 25.30$ mmol, in $20 \mathrm{ml}$ carbon disulfide) was added dropwise over $48 \mathrm{~min}$. After stirring for $30 \mathrm{~min}$. at $0^{\circ} \mathrm{C}$ the reaction contents were filtered through a glass-fritted(fine) büchner funnel, washed with cold carbon disulfide then cold diethyl ether. The tan powder was dried for $10 \mathrm{~min}$ by vacuum suction and used as-is (A), $7.968 \mathrm{~g}(23.57 \mathrm{mmol}), 83.8 \%$.

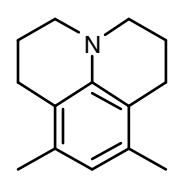

3,5-dimethyljulolidine (B). 3,5-Dimethylaniline $\quad(4.019$ g, 33.17 mmol), 1-bromo-3chloropropane (113.567 g, $721.34 \mathrm{mmol})$, sodium carbonate (14.576 g, $137.95 \mathrm{mmol}$, oven dried at $300^{\circ} \mathrm{C}$ for 4 hours $)$ and $4 \AA$ molecular sieves $\left(3.5 \mathrm{~g}\right.$ activated at $\left.300^{\circ} \mathrm{C}\right)$ were added to a flamedried $250 \mathrm{ml}$ round bottom flask under a nitrogen atmosphere. The following heating rate was applied: $1 \mathrm{hr}$. at $80^{\circ} \mathrm{C}, 2 \mathrm{hr}$. at $100^{\circ} \mathrm{C}, 12 \mathrm{hr}$. at $160^{\circ} \mathrm{C}$. The reaction mixture was cooled to room temperature, diluted with $200 \mathrm{ml}$ methylene chloride, then washed successively with: $500 \mathrm{ml}$ of $10 \% \mathrm{HCl}$, twice with $500 \mathrm{ml}$ water, once with $500 \mathrm{ml}$ of $10 \% \mathrm{NaOH}$ and twice with $500 \mathrm{ml}$ water. The organic fraction was dried over sodium sulfate and then flash column 
chromatographed on silica gel using a solvent gradient beginning with $40 \%$ hexanes in methylene chloride (v/v) and ending with $100 \%$ methylene chloride providing (B) as a white crystalline powder, $5.669 \mathrm{~g}(28.16 \mathrm{mmol}), 84.9 \% . \mathrm{C}_{14} \mathrm{H}_{19} \mathrm{~N}$ HRMS-ESI (m/z): [M+e] 201.1517 (calc. 201.1517). ${ }^{1} \mathrm{H}$ NMR $\left(\mathrm{CDCl}_{3}\right) \delta: 6.380(\mathrm{~s}, 1 \mathrm{H}), 3.065(\mathrm{t}, \mathrm{J}=5.5 \mathrm{~Hz}, 4 \mathrm{H}), 2.630(\mathrm{t}, \mathrm{J}=6.8$ $\mathrm{Hz}, 4 \mathrm{H}), 2.120(\mathrm{~s}, 6 \mathrm{H}), 2.020(\mathrm{~m}, 4 \mathrm{H})$.

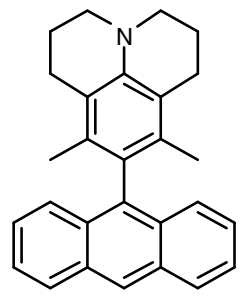

3,5-dimethyl-4-(9-anthracenyl)julolidine (DMJ-An). 9,10-Dibromo-9,10-dihydroanthracene $(1.423 \mathrm{~g}, 4.21 \mathrm{mmol})$ was placed in a flame-dried $100 \mathrm{ml}$ round bottom flask with $20 \mathrm{ml}$ dry chloroform (ethanol free). The reaction flask was cooled to $0^{\circ} \mathrm{C}$ using a water / ice bath under a nitrogen atmosphere. Compound $\mathbf{B}(1.643 \mathrm{~g}, 8.17 \mathrm{mmol}$, in $10 \mathrm{ml}$ dry chloroform) was added dropwise over $35 \mathrm{~min}$. to the cooled reaction mixture. After the addition was complete the reaction was stirred at $0^{\circ} \mathrm{C}$ for an additional 2.5 hours and then warmed to room temperature overnight with continuous stirring under nitrogen. The solvent was removed on a rotary evaporator and the residue purified via flash column chromatography on silica gel. Elution with $40 \%$ hexanes in methylene chloride (v/v) yielded (DMJ-An) as a yellowish powder, $0.301 \mathrm{~g}$ (0.080 mmol), 18.9\%. $\mathrm{C}_{28} \mathrm{H}_{27} \mathrm{~N}$ HRMS-ESI (m/z): $[\mathrm{M}+\mathrm{H}]^{+} 378.2219$ (calc. 378.2222). ${ }^{1} \mathrm{H}$ NMR $\left(\mathrm{CDCl}_{3}\right) \delta: 8.448(\mathrm{~s}, 1 \mathrm{H}), 8.044(\mathrm{~d}, \mathrm{~J}=8.5 \mathrm{~Hz}, 2 \mathrm{H}), 7.593(\mathrm{~d}, \mathrm{~J}=8.7 \mathrm{~Hz}, 2 \mathrm{H}), 7.437(\mathrm{t}, \mathrm{J}=8.5$ $\mathrm{Hz}, 2 \mathrm{H}), 7.315(\mathrm{~m}, 2 \mathrm{H}), 3.180(\mathrm{t}, \mathrm{J}=5.5 \mathrm{~Hz}, 4 \mathrm{H}), 2.732(\mathrm{t}, \mathrm{J}=6.8 \mathrm{~Hz}, 4 \mathrm{H}), 2.120(\mathrm{~m}, 4 \mathrm{H}), 1.510$ 
$(\mathrm{s}, 6 \mathrm{H})$.

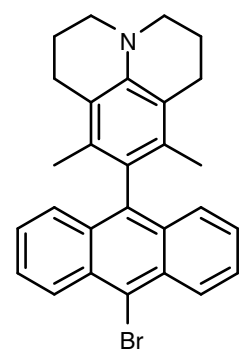

3,5-dimethyl-4-(10-bromoanthracenyl)julolidine (C). DMJ-An $(0.139 \mathrm{~g}, 0.369 \mathrm{mmol})$ was added to a $50 \mathrm{ml}$ round bottom flask with $20 \mathrm{ml}$ acetic acid. Sonication and slight heating was necessary to fully dissolve the starting material. Using a disposable Pasteur pipette, seven drops of bromine were added slowly. There was an immediate color change to dark brown and a slight precipitate. Continued stirring for an additional 15 minutes yielded a light tan solution. The reaction mixture was diluted with methylene chloride and washed twice with water and then once with a $\mathrm{NaHCO}_{3}$ solution. Purification via a plug of silica gel using methylene chloride (v/v) provided (C) as a yellowish solid, $0.100 \mathrm{~g}(0.219 \mathrm{mmol}), 60.0 \%$. $\mathrm{C}_{28} \mathrm{H}_{26} \mathrm{BrN}$ HRMS-ESI (m/z): $[\mathrm{M}+\mathrm{H}]^{+} 456.1305$ (calc. 456.1327). ${ }^{1} \mathrm{H} \mathrm{NMR}\left(\mathrm{CDCl}_{3}\right) \delta: 8.585(\mathrm{~d}, \mathrm{~J}=8.8 \mathrm{~Hz}, 2 \mathrm{H}), 7.693-$ $7.535(\mathrm{~m}, 4 \mathrm{H}), 7.343(\mathrm{dd}, \mathrm{J}=8.7 \mathrm{~Hz}, 2 \mathrm{H}), 3.181(\mathrm{t}, \mathrm{J}=5.5 \mathrm{~Hz}, 4 \mathrm{H}), 2.712(\mathrm{t}, \mathrm{J}=6.8 \mathrm{~Hz}, 4 \mathrm{H})$, $2.113(\mathrm{~m}, 4 \mathrm{H}), 1.486(\mathrm{~s}, 6 \mathrm{H})$.

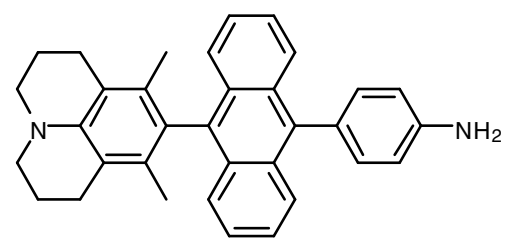

3,5-dimethyl-4-(10-(4-aminophenyl)anthracenyl)julolidine (D). Compound C (0.050 g, 0.110 mmol), 4-(4,4,5,5-tetramethyl-1,3,2-dioxaborolyl)aminobenzene $(0.043 \mathrm{~g}, 0.196 \mathrm{mmol})$ and 
palladium (0) tetrakistriphenylphosphine $(0.005 \mathrm{~g}, 0.004 \mathrm{mmol})$ were combined in a $25 \mathrm{ml}$ round bottom flask with $8 \mathrm{ml}$ toluene. Sodium carbonate $(0.034 \mathrm{~g}, 0.321 \mathrm{mmol})$ was added as an aqueous solution $(1 \mathrm{ml})$ and the contents degassed with nitrogen for $5 \mathrm{~min}$. The contents were heated to $85^{\circ} \mathrm{C}$ under a nitrogen atmosphere with the exclusion of light for a total of 35 hrs. Additional 4-(4,4,5,5-tetramethyl-1,3,2-dioxaborolyl)aminobenzene (0.055 g, $0.251 \mathrm{mmol})$ and palladium (0) tetrakistriphenylphosphine $(0.005 \mathrm{~g}, 0.004 \mathrm{mmol})$ were added after $7 \mathrm{hrs}$. The reaction contents were cooled to room temperature, diluted with methylene chloride, washed twice with water and dried over sodium sulfate. Purification required (2) successive silica flash chromatography columns. The first was eluted with a solvent gradient from $40 \%$ hexanes in methylene chloride (v/v) to $100 \%$ methylene chloride. The second was eluted with a solvent gradient from $100 \%$ methylene chloride to $5 \%$ acetone in methylene chloride $(\mathrm{v} / \mathrm{v})$ to provide (D) as a yellowish powder, $0.036 \mathrm{~g}(0.077 \mathrm{mmol}), 70.1 \% . \mathrm{C}_{34} \mathrm{H}_{32} \mathrm{~N}_{2} \mathrm{HRMS}-\mathrm{ESI}(\mathrm{m} / \mathrm{z})$ : $[\mathrm{M}+\mathrm{H}]^{+}$ 469.2632 (calc. 469.2644). ${ }^{1} \mathrm{H} \mathrm{NMR}\left(\mathrm{CDCl}_{3}\right) \delta: 7.813(\mathrm{~d}, \mathrm{~J}=8.8 \mathrm{~Hz}, 2 \mathrm{H}), 7.620(\mathrm{~d}, \mathrm{~J}=7.6 \mathrm{~Hz}$, 2H), $7.350-7.256(\mathrm{~m}, 6 \mathrm{H}), 6.935(\mathrm{~d}, \mathrm{~J}=8.3 \mathrm{~Hz}, 2 \mathrm{H}), 3.845(\mathrm{~s}, 2 \mathrm{H}), 3.188(\mathrm{t}, \mathrm{J}=5.5 \mathrm{~Hz}, 4 \mathrm{H})$, $2.741(\mathrm{t}, \mathrm{J}=6.8 \mathrm{~Hz}, 4 \mathrm{H}), 2.125(\mathrm{~m}, 4 \mathrm{H}), 1.564(\mathrm{~s}, 6 \mathrm{H})$.

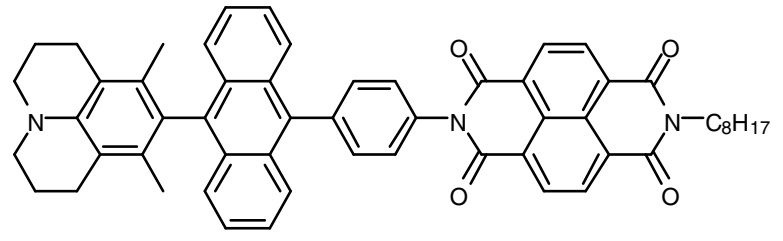

DMJ-An-Ph $-\mathbf{N I}$ (1). Compound D (0.030 g, $0.064 \mathrm{mmol})$ and $N$-(n-octyl)-naphthalene-1,8dicarboxyanhydride-4,5-dicarboximide $(0.055 \mathrm{~g}, 0.145 \mathrm{mmol})$ were added to a flame-dried $25 \mathrm{ml}$ round bottom flask and $10 \mathrm{ml}$ dry pyridine added. The reaction mixture was heated to $110^{\circ} \mathrm{C}$ 
under a nitrogen atmosphere for 11 hrs. Additional $N$-(n-octyl)-naphthalene-1,8dicarboxyanhydride-4,5-dicarboximide $(0.050 \mathrm{~g}, 0.132 \mathrm{mmol})$ was added after $7 \mathrm{hrs}$. Upon completion, the pyridine was removed on a rotary evaporator and the crude product purified via flash column chromatography. Elution with a solvent gradient of $100 \%$ methylene chloride to $5 \%$ acetone in methylene chloride (v/v) provided (1) as a yellowish powder, $0.028 \mathrm{~g}(0.034$ mmol), 52.7\%. $\mathrm{C}_{56} \mathrm{H}_{51} \mathrm{~N}_{3} \mathrm{O}_{4}$ HRMS-ESI (m/z): $[\mathrm{M}+\mathrm{H}]^{+} 830.3958$ (calc. 830.3958). ${ }^{1} \mathrm{H}$ NMR $\left(\mathrm{CDCl}_{3}\right) \delta: 8.884(\mathrm{dd}, \mathrm{J}=7.5 \mathrm{~Hz}, 4 \mathrm{H}), 7.834(\mathrm{~d}, \mathrm{~J}=8.7 \mathrm{~Hz}, 2 \mathrm{H}), 7.724(\mathrm{~d}, \mathrm{~J}=8.3 \mathrm{~Hz}, 2 \mathrm{H})$, $7.672(\mathrm{~d}, \mathrm{~J}=8.2 \mathrm{~Hz}, 2 \mathrm{H}), 7.575(\mathrm{~d}, \mathrm{~J}=8.3 \mathrm{~Hz}, 2 \mathrm{H}), 7.443-7.291(\mathrm{~m}, 4 \mathrm{H}), 4.242(\mathrm{t}, \mathrm{J}=7.7 \mathrm{~Hz}$, 2H), $3.201(\mathrm{t}, \mathrm{J}=5.5 \mathrm{~Hz}, 4 \mathrm{H}), 2.756(\mathrm{t}, \mathrm{J}=6.8 \mathrm{~Hz}, 4 \mathrm{H}), 2.145(\mathrm{~m}, 4 \mathrm{H}), 1.790,(\mathrm{~m}, 2 \mathrm{H}), 1.591(\mathrm{~s}$, 6H), 1.299 (br. s, 10H), $0.892(\mathrm{t}, \mathrm{J}=6.7 \mathrm{~Hz}, 3 \mathrm{H})$.

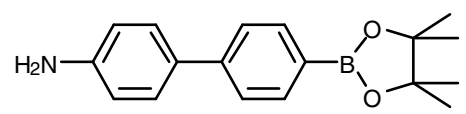

4-amino-4'-(4,4,5,5-tetramethyl-1,3,2-dioxaborolyl)biphenyl (E). 4-Amino-4'-bromobiphenyl $(0.507 \mathrm{~g}, 2.04 \mathrm{mmol})$, palladium (II) acetate $(0.026 \mathrm{~g}, 0.12 \mathrm{mmol})$, potassium acetate $(0.592 \mathrm{~g}$, $6.03 \mathrm{mmol})$ and bis(pinacolato)diboron $(0.517 \mathrm{~g}, 2.04 \mathrm{mmol})$ were combined in a $10 \mathrm{ml}$ round bottom flask with $4 \mathrm{ml}$ dry DMF. Nitrogen was bubbled through the solution for 10 min to degas, then the contents heated to $85^{\circ} \mathrm{C}$ for 3.5 hours. The reaction contents were diluted with methylene chloride and washed with water to remove excess potassium acetate and DMF. Flash column chromatography on silica using methylene chloride as the eluent provided (E) as a yellowish solid, $0.319 \mathrm{~g}$ (1.08 mmol), $52.9 \% . \mathrm{C}_{18} \mathrm{H}_{22} \mathrm{BNO}_{2} \mathrm{HRMS}-\mathrm{ESI}(\mathrm{m} / \mathrm{z}):[\mathrm{M}+\mathrm{H}]^{+} 296.1808$ (calc. 296.1822). ${ }^{1} \mathrm{H}$ NMR $500 \mathrm{MHz}\left(\mathrm{CDCl}_{3}\right) \delta: 7.843(\mathrm{~d}, \mathrm{~J}=7.6 \mathrm{~Hz}, 2 \mathrm{H}), 7.558(\mathrm{~d}, \mathrm{~J}=7.6 \mathrm{~Hz}$, 
2H), $7.440(\mathrm{~d}, \mathrm{~J}=8.2 \mathrm{~Hz}, 2 \mathrm{H}), 6.759$ (d, J = 8.2 Hz, 2H), 3.754 (s, 2H), 1.354 (s, 12H).

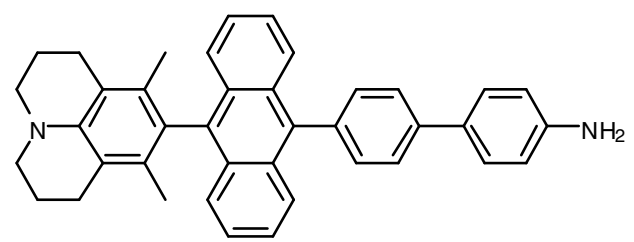

3,5-dimethyl-4-(10-(4'-aminobiphenyl)anthracenyl)julolidine (F). Compound C (0.236 g, $0.52 \mathrm{mmol}$ ) was added to a $25 \mathrm{ml}$ round bottom flask with $10 \mathrm{ml}$ toluene and bubbled with nitrogen for 10 minutes. Palladium (0) tetrakistriphenylphosphine (0.020 g, $0.02 \mathrm{mmol})$ and 4amino-4'-(4,4,5,5-tetramethyl-1,3,2-dioxaborolyl)biphenyl $(0.174 \mathrm{~g}, 0.59 \mathrm{mmol})$ were then added to the reaction flask. Sodium carbonate $(0.315 \mathrm{~g}, 2.97 \mathrm{mmol}$ in $1.5 \mathrm{ml}$ water $)$ was added and the reaction mixture heated to $95^{\circ} \mathrm{C}$ under nitrogen with the exclusion of light for 5 hours. The mixture was diluted with dichloromethane, washed with water and dried. Flash column chromatography on silica gel using $2 \%$ acetone in dichloromethane (v/v) as the eluent provided (F) as a yellow powder, $0.120 \mathrm{~g}(0.22 \mathrm{mmol}), 42.6 \% . \quad \mathrm{C}_{40} \mathrm{H}_{36} \mathrm{~N}_{2}$ HRMS-ESI (m/z): [M+H] $]^{+}$ 545.2930 (calc. 545.2957). ${ }^{1} \mathrm{H}$ NMR $500 \mathrm{MHz}\left(\mathrm{CDCl}_{3}\right) \delta: 7.788(\mathrm{~m}, 4 \mathrm{H}), 7.626(\mathrm{~m}, 4 \mathrm{H}), 7.541$ $(\mathrm{d}, \mathrm{J}=8.0 \mathrm{~Hz}, 2 \mathrm{H}), 7.317(\mathrm{~m}, 4 \mathrm{H}), 6.852(\mathrm{~d}, \mathrm{~J}=8.3 \mathrm{~Hz}, 2 \mathrm{H}), 3.804(\mathrm{~s}, 2 \mathrm{H}), 3.195$ (t, J = 5.0 Hz, 4H), $2.751(\mathrm{t}, \mathrm{J}=6.5 \mathrm{~Hz}, 4 \mathrm{H}), 2.138(\mathrm{~m}, 4 \mathrm{H}), 1.582(\mathrm{~s}, 6 \mathrm{H})$.

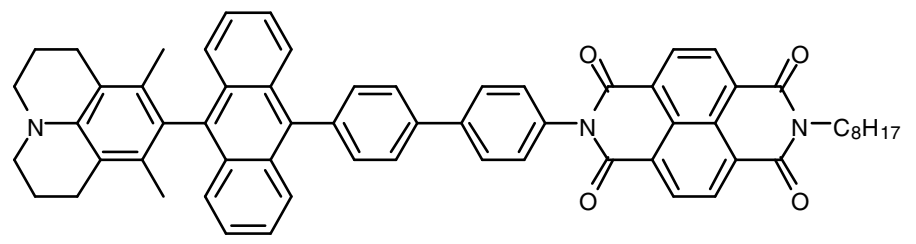

DMJ-An-Ph $-\mathbf{N I}$ (2). Compound F (0.028 g, $0.05 \mathrm{mmol})$ and $N$-(n-octyl)-naphthalene-1,8dicarboxyanhydride-4,5-dicarboximide $^{1}(0.060 \mathrm{~g}, 0.16 \mathrm{mmol})$ were added to a $10 \mathrm{ml}$ round 
bottom flask with $2 \mathrm{ml}$ dry pyridine. The contents were heated to reflux under nitrogen for 7 hours. The reaction mixture was then cooled to room temperature and the pyridine removed on a rotary evaporator to give a tan colored solid. Purification was performed on a silica column using neat dichloromethane as the eluent to give (2) $0.027 \mathrm{~g}(0.03 \mathrm{mmol}), 58 \% . \mathrm{C}_{62} \mathrm{H}_{55} \mathrm{~N}_{3} \mathrm{O}_{4}$ HRMS-ESI (m/z): [M+e] 905.4195 (calc. 905.4193). ${ }^{1} \mathrm{H}$ NMR $500 \mathrm{MHz}\left(\mathrm{CDCl}_{3}\right) \delta: 8.866(\mathrm{dd}$, $\mathrm{J}=7.5 \mathrm{~Hz}, 4 \mathrm{H}), 7.993(\mathrm{~d}, \mathrm{~J}=8.3 \mathrm{~Hz}, 2 \mathrm{H}), 7.914(\mathrm{~d}, \mathrm{~J}=8.0 \mathrm{~Hz}, 2 \mathrm{H}), 7.809(\mathrm{~d}, \mathrm{~J}=8.5 \mathrm{~Hz}, 2 \mathrm{H})$, $7.674(\mathrm{~d}, \mathrm{~J}=8.5 \mathrm{~Hz}, 2 \mathrm{H}), 7.651(\mathrm{~d}, \mathrm{~J}=8.0 \mathrm{~Hz}, 2 \mathrm{H}), 7.508(\mathrm{~d}, \mathrm{~J}=8.3 \mathrm{~Hz}, 2 \mathrm{H}), 7.41-7.31(\mathrm{~m}$, 4H), $4.247(\mathrm{t}, \mathrm{J}=7.7 \mathrm{~Hz}, 2 \mathrm{H}), 3.214(\mathrm{t}, \mathrm{J}=5.0 \mathrm{~Hz}, 4 \mathrm{H}), 2.771(\mathrm{t}, \mathrm{J}=6.5 \mathrm{~Hz}, 4 \mathrm{H}), 2.156(\mathrm{~m}, 4 \mathrm{H})$, $1.793(\mathrm{~m}, 2 \mathrm{H}), 1.607$ (s, 6H), 1.309 (br. s, 10H), 0.904 (t, J = 6.8 Hz, 3H).

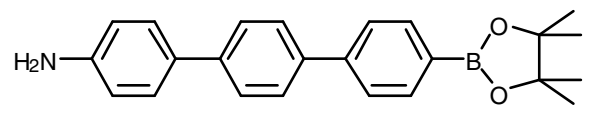

\section{4-amino-4"'-(4,4,5,5-tetramethyl-1,3,2-dioxaborolyl)terphenyl $\quad$ (G). 4-Amino-4"'-} bromoterphenyl $(0.510 \mathrm{~g}, 1.57 \mathrm{mmol})$, palladium (II) acetate $(0.023 \mathrm{~g}, 0.10 \mathrm{mmol})$, potassium acetate $(0.455 \mathrm{~g}, 4.64 \mathrm{mmol})$ and bis(pinacolato)diboron $(0.399 \mathrm{~g}, 1.57 \mathrm{mmol})$ were combined in a flame-dried $10 \mathrm{ml}$ round bottom flask with $3 \mathrm{ml}$ dry DMF. The solution was bubbled with nitrogen for $10 \mathrm{~min}$ to remove oxygen then heated to $90^{\circ} \mathrm{C}$ for 7 hours. The reaction mixture was diluted with methylene chloride and washed with water to remove DMF and potassium acetate. Column chromatography on silica using $1 \%$ acetone in methylene chloride (v/v) as the eluent gave (G) $0.510 \mathrm{~g}(1.37 \mathrm{mmol}), 84.4 \% . \mathrm{C}_{24} \mathrm{H}_{26} \mathrm{BNO}_{2}$ HRMS-ESI (m/z): [M+e] 371.2055 (calc. 371.2057). ${ }^{1} \mathrm{H}$ NMR $500 \mathrm{MHz}\left(\mathrm{CDCl}_{3}\right)$ 8: $7.900(\mathrm{~d}, \mathrm{~J}=7.7 \mathrm{~Hz}, 2 \mathrm{H}), 7.655$ (m, 4H), 7.619 $(\mathrm{d}, \mathrm{J}=8.1 \mathrm{~Hz}, 2 \mathrm{H}), 7.474(\mathrm{~d}, \mathrm{~J}=8.1 \mathrm{~Hz}, 2 \mathrm{H}), 6.848(\mathrm{~d}, \mathrm{~J}=7.7 \mathrm{~Hz}, 2 \mathrm{H}), 4.890(\mathrm{~s}, 2 \mathrm{H}), 1.369(\mathrm{~s}$, 
12H).

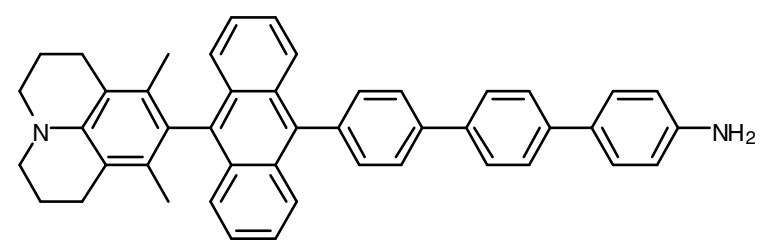

\section{3,5-dimethyl-4-(10-(4"'-aminoterphenyl)anthracenyl)julolidine (H)}

Compound $\mathbf{C}(0.229 \mathrm{~g}, 0.51 \mathrm{mmol})$ was added to a $25 \mathrm{ml}$ round bottom flask with $5 \mathrm{ml}$ toluene and bubbled with nitrogen for 10 minutes. Palladium (0) tetrakistriphenylphosphine $(0.025 \mathrm{~g}$, $0.02 \mathrm{mmol})$ and $\mathbf{G}(0.230 \mathrm{~g}, 0.62 \mathrm{mmol})$ were then added to the reaction flask. Sodium carbonate $(0.263 \mathrm{~g}, 2.48 \mathrm{mmol}$ in $1 \mathrm{ml}$ water $)$ was added and the reaction mixture heated to $90^{\circ}$ C under nitrogen with the exclusion of light for 3 hours. The mixture was diluted with dichloromethane, washed with water and dried. Flash column chromatography on silica gel using $1.5 \%$ acetone in dichloromethane (v/v) as the eluent provided $(\mathbf{H}) 0.170 \mathrm{~g}(0.27 \mathrm{mmol})$, $54.6 \% . \quad \mathrm{C}_{46} \mathrm{H}_{40} \mathrm{~N}_{2} \quad$ HRMS-ESI (m/z): [M+e] 620.3193 (calc. 620.3192). ${ }^{1} \mathrm{H}$ NMR $500 \mathrm{MHz}$ $\left(\mathrm{CDCl}_{3}\right) \delta: 7.816(\mathrm{~m}, 4 \mathrm{H}), 7.712(\mathrm{~d}, \mathrm{~J}=8.5 \mathrm{~Hz}, 2 \mathrm{H}), 7.640(\mathrm{~m}, 4 \mathrm{H}), 7.591(\mathrm{~d}, \mathrm{~J}=8.5 \mathrm{~Hz}, 2 \mathrm{H})$ $7.532(\mathrm{~d}, \mathrm{~J}=8.5 \mathrm{~Hz}, 2 \mathrm{H}), 7.329(\mathrm{~m}, 4 \mathrm{H}), 6.813(\mathrm{~d}, \mathrm{~J}=8.5 \mathrm{~Hz}, 2 \mathrm{H}), 3.756(\mathrm{~s}, 2 \mathrm{H}), 3.194(\mathrm{t}, \mathrm{J}=$ $5.2 \mathrm{~Hz}, 4 \mathrm{H}), 2.945$ (m, 4H), 2.749 (t, J = 6.1 Hz, 4H), 1.585 (s, 6H).

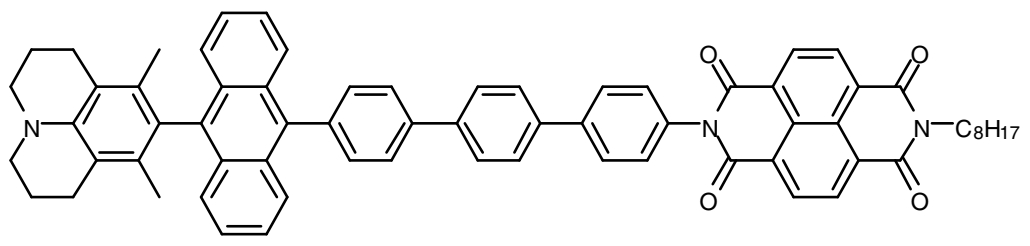

\section{DMJ-An-Ph ${ }_{3}$-NI (3)}

Compound $\mathbf{H}(0.070 \mathrm{~g}, 0.11 \mathrm{mmol})$ and $N$-(n-octyl)-naphthalene-1,8-dicarboxyanhydride-4,5- 
dicarboximide $^{1}(0.090 \mathrm{~g}, 0.24 \mathrm{mmol})$ were added to a $25 \mathrm{ml}$ round bottom flask with $4 \mathrm{ml}$ dry pyridine. The contents were heated to reflux under nitrogen for 10 hours. The reaction mixture was then cooled to room temperature and the pyridine removed on a rotary evaporator to give a tan colored solid. Purification was performed on a silica column using $2 \%$ acetone in dichloromethane $(\mathrm{v} / \mathrm{v})$ as the eluent to give (3) $0.062 \mathrm{~g}(0.04 \mathrm{mmol}), 55.5 \% . \quad \mathrm{C}_{68} \mathrm{H}_{59} \mathrm{~N}_{3} \mathrm{O}_{4}$ HRMS-ESI (m/z): [M+e] 981.4509 (calc. 981.4506). ${ }^{1} \mathrm{H}$ NMR $500 \mathrm{MHz}\left(\mathrm{CDCl}_{3}\right) \delta: 8.846(\mathrm{dd}, \mathrm{J}$ $=7.5 \mathrm{~Hz}, 4 \mathrm{H}), 7.904(\mathrm{~m}, 6 \mathrm{H}), 7.829(\mathrm{~d}, \mathrm{~J}=8.1 \mathrm{~Hz}, 2 \mathrm{H}), 7.806(\mathrm{~d}, \mathrm{~J}=8.5 \mathrm{~Hz}, 2 \mathrm{H}), 7.658(\mathrm{~d}, \mathrm{~J}=$ $8.5 \mathrm{~Hz}, 2 \mathrm{H}), 7.633(\mathrm{~d}, \mathrm{~J}=8.0 \mathrm{~Hz}, 2 \mathrm{H}), 7.463(\mathrm{~d}, \mathrm{~J}=8.2 \mathrm{~Hz}, 2 \mathrm{H}), 7.4-7.3(\mathrm{~m}, 4 \mathrm{H}), 4.229(\mathrm{t}, \mathrm{J}=$ $7.5 \mathrm{~Hz}, 2 \mathrm{H}), 3.199(\mathrm{t}, \mathrm{J}=5.3 \mathrm{~Hz}, 4 \mathrm{H}), 2.756(\mathrm{t}, \mathrm{J}=6.5 \mathrm{~Hz}, 4 \mathrm{H}), 2.143(\mathrm{~m}, 4 \mathrm{H}), 1.777(\mathrm{~m}, 2 \mathrm{H})$, 1.593 (s, 6H), 1.294 (br. s, 10H), 0.889 (t, J = 6.8 Hz, 3H).

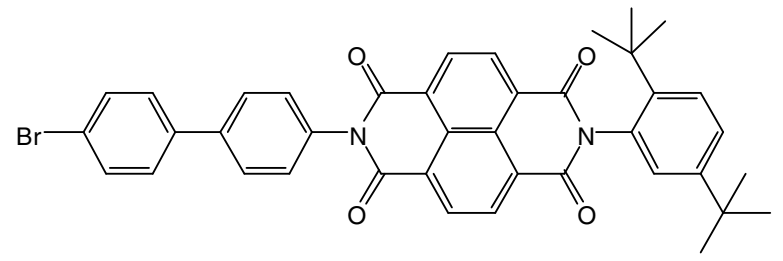

\section{4-Bromobiphenyl)-NI (I)}

$N$-(2,5-di-t-butylphenyl)-naphthalene-1,8-dicarboxyanhydride-4,5-dicarboximide (1.65 g, 3.63 mmol) and 4-amino-4'-bromobiphenyl (1.08 g, $4.40 \mathrm{mmol})$ were added to a $100 \mathrm{ml}$ round bottom flask with $40 \mathrm{ml}$ of dry pyridine. The reaction mixture was heated to reflux under nitrogen and without the presence of light for 15 hours. The reaction mixture was cooled to room temperature and the solvents were removed by rotary evaporation. Purification was performed on a silica column using methylene chloride as the eluent to give (I) $1.75 \mathrm{~g}$ (2.55 mmol), $71 \% . \mathrm{C}_{40} \mathrm{H}_{33} \mathrm{BrN}_{2} \mathrm{O}_{4}$ HRMS-ESI (m/z): $[\mathrm{M}+\mathrm{H}]^{+} 685.1721$ (calc. 685.1702). NMR 500 
$\mathrm{MHz}\left(\mathrm{CDCl}_{3}\right)$ 8: $8.812(\mathrm{~s}, 4 \mathrm{H}), 7.699(\mathrm{~d}, \mathrm{~J}=8.5 \mathrm{~Hz}, 2 \mathrm{H}), 7.521(\mathrm{~d}, \mathrm{~J}=8.0 \mathrm{~Hz}, 2 \mathrm{H}), 7.443(\mathrm{dd}, \mathrm{J}$ $=8.2 \mathrm{~Hz}, 4 \mathrm{H}), 7.355(\mathrm{~d}, 2 \mathrm{H}), 6.955(\mathrm{~s}, 1 \mathrm{H}), 1.287(\mathrm{~s}, 9 \mathrm{H}), 1.201(\mathrm{~s}, 9 \mathrm{H})$.

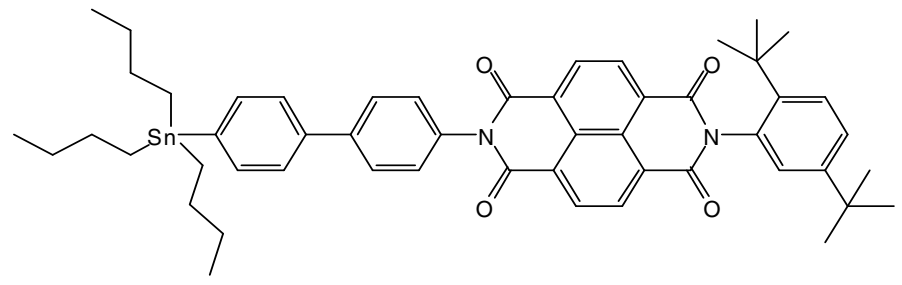

4-(tri-n-butylstannyl)biphenyl-NI (J). Compound I (1.88 g, $2.75 \mathrm{mmol})$ was added to a $200 \mathrm{ml}$ round bottom flask with $80 \mathrm{ml}$ dry toluene. Bis(tributyltin) $(6.86 \mathrm{ml}, 13.7 \mathrm{mmol})$ and Palladium (0) tetrakistriphenylphosphine were added and the contents were set to heat at $100{ }^{\circ} \mathrm{C}$, without the presence of light, for 16 hours. The reaction mixture was cooled to room temperature and the solvent was removed by rotary evaporation. Purification was performed on a silica column using methylene chloride as the eluent to give (J) $1.325 \mathrm{~g}(1.48 \mathrm{mmol}), 54 \% . \mathrm{C}_{52} \mathrm{H}_{60} \mathrm{~N}_{2} \mathrm{O}_{4} \mathrm{Sn}$ HRMSESI (m/z): $[\mathrm{M}+\mathrm{H}]^{+} 897.3614$ (calc. 897.3653). NMR $500 \mathrm{MHz}\left(\mathrm{CDCl}_{3}\right)$ ) 8.797 (s, 4H), 7.020 (m, 2H), $7.540(\mathrm{~m}, 3 \mathrm{H}), 7.433(\mathrm{~m}, 3 \mathrm{H}), 7.340(\mathrm{~d}, \mathrm{~J}=8.27 \mathrm{~Hz}, 2 \mathrm{H}), 6.954(\mathrm{~s}, 1 \mathrm{H}), 1.490(\mathrm{~m}, 6 \mathrm{H})$, $1.300(\mathrm{~m}, 6 \mathrm{H}), 1.260(\mathrm{~s}, 9 \mathrm{H}), 1.208(\mathrm{~s}, 9 \mathrm{H}), 1.030(\mathrm{~m}, 6 \mathrm{H}), 0.839$ (t, J=7.31 Hz, 9).

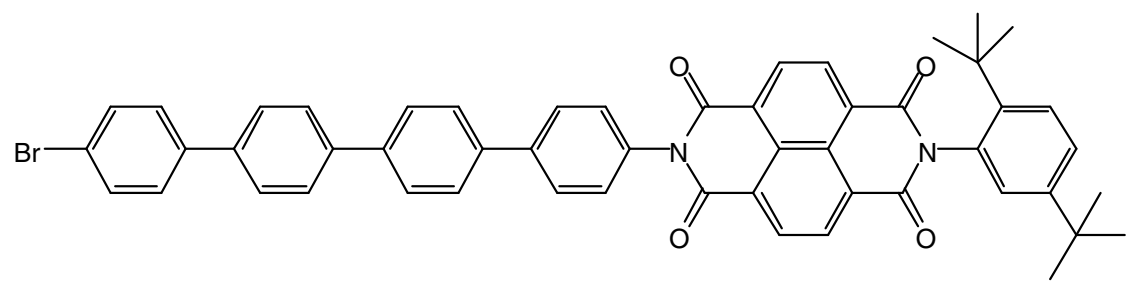

4"'-Bromoquaterphenyl-NI (K). Stannane J (0.378 g, $0.42 \mathrm{mmol})$ and 4,4'-dibromobiphenyl $(0.40 \mathrm{~g}, 1.27 \mathrm{mmol})$ were added to a $50 \mathrm{ml}$ round bottom flask with $20 \mathrm{ml}$ of dry toluene. 
Palladium (0) tetrakistriphenylphosphine was added and the contents were set to heat at $100{ }^{\circ} \mathrm{C}$, without the presence of light, for 14 hours. The reaction mixture was cooled to room temperature and the solvent was removed using rotary evaporation. Purification was performed on a silica column using methylene chloride as the eluent to give $0.105 \mathrm{~g}(0.125 \mathrm{mmol}), 30 \%$ of the yellow (K). $\mathrm{C}_{52} \mathrm{H}_{41} \mathrm{BrN}_{2} \mathrm{O}_{4}$ HRMS-FAB (m/z): [M] ${ }^{+} 836.2247$ (calc. 836.2250) NMR 500 $\mathrm{MHz}\left(\mathrm{CDCl}_{3}\right) \delta: 8.879(\mathrm{~d}, \mathrm{~J}=7.5 \mathrm{~Hz}, 4 \mathrm{H}), 7.9(\mathrm{~m}, 4 \mathrm{H}), 7.848(\mathrm{~d}, \mathrm{~J}=8.0 \mathrm{~Hz}, 2 \mathrm{H}), 7.8(\mathrm{~m}, 2 \mathrm{H})$, $7.638(\mathrm{~d}, \mathrm{~J}=8.0 \mathrm{~Hz}, 2 \mathrm{H}), 7.543(\mathrm{dt}, \mathrm{J}=8.2 \mathrm{~Hz}, 2 \mathrm{H}), 7.481(\mathrm{~d}, \mathrm{~J}=7.9 \mathrm{~Hz}, 2 \mathrm{H})$, 7.4-7.26 (m, 4H), $7.096(\mathrm{~s}, 1 \mathrm{H}), 1.287(\mathrm{~s}, 9 \mathrm{H}), 1.201(\mathrm{~s}, 9 \mathrm{H})$.

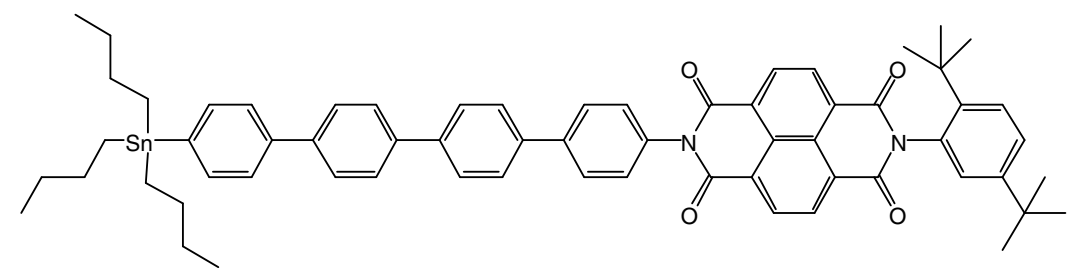

4"'-(tri-n-butylstannyl)quaterphenyl-NI (L). Compound K (0.105 g, $0.126 \mathrm{mmol})$ and bis(tributyltin) $(0.38 \mathrm{ml}, 0.756 \mathrm{mmol})$ were added to a $25 \mathrm{ml}$ round bottom flask with $10 \mathrm{ml}$ of dry toluene. Palladium (0) tetrakistriphenylphosphine was added and the contents were set to heat at $100{ }^{\circ} \mathrm{C}$, without the presence of light, for 48 hours. The reaction mixture was cooled to room temperature and the solvent was removed by rotary evaporation. Purification was performed on a silica column using methylene chloride as the eluent to give (L) $0.048 \mathrm{~g}(0.046$ mmol), 37\%. $\mathrm{C}_{64} \mathrm{H}_{68} \mathrm{~N}_{2} \mathrm{O}_{4} \mathrm{Sn} \quad$ LRMS-FAB (m/z): $[\mathrm{M}]^{+}$1047.1 (calc. 1048.4) NMR $500 \mathrm{MHz}$ $\left(\mathrm{CDCl}_{3}\right) \delta: 8.881(\mathrm{~d}, \mathrm{~J}=7.5 \mathrm{~Hz}, 4 \mathrm{H}), 7.9(\mathrm{~m}, 4 \mathrm{H}), 7.848(\mathrm{~d}, \mathrm{~J}=8.0 \mathrm{~Hz}, 2 \mathrm{H}), 7.8(\mathrm{~m}, 2 \mathrm{H}), 7.638$ $(\mathrm{d}, \mathrm{J}=8.0 \mathrm{~Hz}, 2 \mathrm{H}), 7.543(\mathrm{dt}, \mathrm{J}=8.2 \mathrm{~Hz}, 2 \mathrm{H}), 7.481(\mathrm{~d}, \mathrm{~J}=7.9 \mathrm{~Hz}, 2 \mathrm{H}), \quad 7.4-7.26(\mathrm{~m}, 4 \mathrm{H})$, 
$7.096(\mathrm{~s}, 1 \mathrm{H}), 1.6(\mathrm{~m}, 12 \mathrm{H}), 1.25(\mathrm{~m}, 24 \mathrm{H}), 0.895(\mathrm{~m}, 9 \mathrm{H})$.

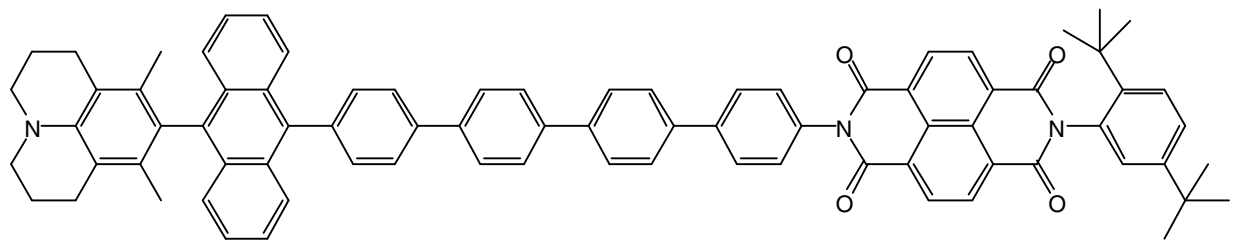

DMJ-An-Ph 4 -NI (4). Stannane L (0.048 g, $0.046 \mathrm{mmol})$ and compound C (0.063 g, 0.014 mmol) were added to a $25 \mathrm{ml}$ round bottom flask with $10 \mathrm{ml}$ of dry toluene. Palladium (0) tetrakistriphenylphosphine was added and the contents were allowed to heat at $100{ }^{\circ} \mathrm{C}$ overnight, without the presence of light. The reaction mixture was cooled to room temperature and the solvent was removed by rotary evaporation. Purification was performed on a silica column using methylene chloride as the eluent to give (4) $0.006 \mathrm{~g}(0.005 \mathrm{mmol}), 40 \% . \mathrm{C}_{80} \mathrm{H}_{67} \mathrm{~N}_{3} \mathrm{O}_{4}$ HRMSFAB (m/z): $[\mathrm{M}]^{+} 1133.5127$ (calc. 1133.5132) NMR $500 \mathrm{MHz}\left(\mathrm{CDCl}_{3}\right)$ 8: $8.812(\mathrm{dd}, \mathrm{J}=7.5 \mathrm{~Hz}$, 4H), $7.884(\mathrm{dd}, \mathrm{J}=8.1 \mathrm{~Hz}, 2 \mathrm{H}), 7.801(\mathrm{~m}, 3 \mathrm{H}), 7.729(\mathrm{~d}, \mathrm{~J}=8.5 \mathrm{~Hz}, 2 \mathrm{H}), 7.689(\mathrm{~d}, \mathrm{~J}=8.5 \mathrm{~Hz}$, 2H), $7.617(\mathrm{~m}, 2 \mathrm{H}), 7.579(\mathrm{~d}, \mathrm{~J}=8.2 \mathrm{~Hz}, 2 \mathrm{H}), 7.452(\mathrm{dd}, 1 \mathrm{H}), 7.4-7.25(\mathrm{~m}, 12 \mathrm{H}), 6.965(\mathrm{~d}, \mathrm{~J}=$ $7.9 \mathrm{~Hz}, 1 \mathrm{H}), 3.2-3.0(\mathrm{~m}, 2 \mathrm{H}), 2.7-2.65(\mathrm{~m}, 2 \mathrm{H}), 2.1-2.0(\mathrm{~m}, 2 \mathrm{H}), 1.523(\mathrm{~s}, 6 \mathrm{H}), 1.287(\mathrm{~s}, 9 \mathrm{H})$, 1.201 (s, 9H). 


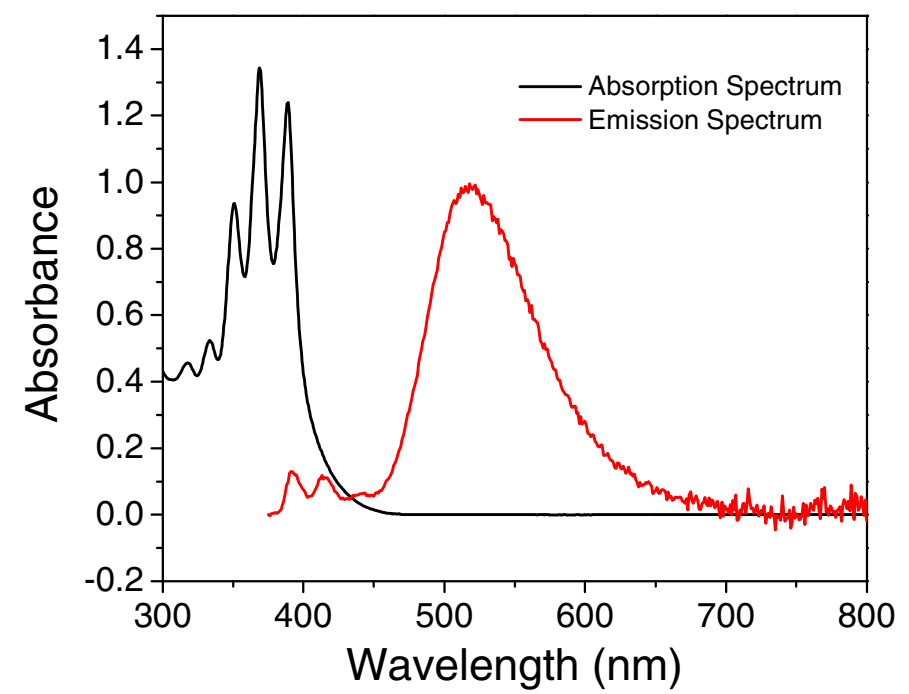

Figure S1. Ground state absorption and fluorescence spectra of the indicated molecules in toluene. The intensity of the fluorescence spectrum is in arbitrary units.

\section{Solvatochromism of DMJ-An}

The excited-state dipole moment $\left(\mu_{\mathrm{e}}\right)$ of DMJ-An was determined by the Lippert-Mataga fluorescence solvatochromic shift method ${ }^{4,5}$ in which the CT emission $\lambda_{\max }$ was measured in six solvents with a range of dielectric constants $(2.02-35.9)$ and plotted against $\mathrm{f}(\varepsilon, \mathrm{n}),[(\varepsilon-1) /(2 \varepsilon+1)$ $\left.-0.5 x\left(n^{2}-1\right) /\left(2 n^{2}+1\right)\right]$, Figure S2. The radius of the Onsager spherical cavity was determined to be $6.5 \AA$ using the $\mathrm{PM} 3^{6}$ optimized DMJ-An structure, which is in agreement with that previously estimated for the non-methylated derivative 4-(9-anthracenyl)-julolidine (J-An). ${ }^{7}$ Using this value and the slope of the Lippert-Mataga plot $(-3.01 \pm 0.21 \mathrm{eV} / \mathrm{f}(\varepsilon, \mathrm{n}))$ the excitedstate dipole moment was found to be $26 \mathrm{D}$. We estimate the degree of charge separation in the CT state as $26 \mathrm{D} / 4.8 \mathrm{D} \mathrm{esu}^{-1} \AA^{-1}=5.4$ esu- $\AA$. Using the centers of the spin density distributions in $\mathrm{DMJ}^{+\bullet}$ and $\mathrm{An}^{-\bullet}, r_{\mathrm{DMJ}-\mathrm{An}}$ for a full charge transferred to yield $\mathrm{DMJ}^{+\bullet}-\mathrm{An}^{-\bullet}$ is $5.6 \AA$. Thus, the estimated percentage of charge separation in the CT state is (5.4 esu- $\AA$ /5.6 esu- $\AA$ ) x $100=96 \%$. 


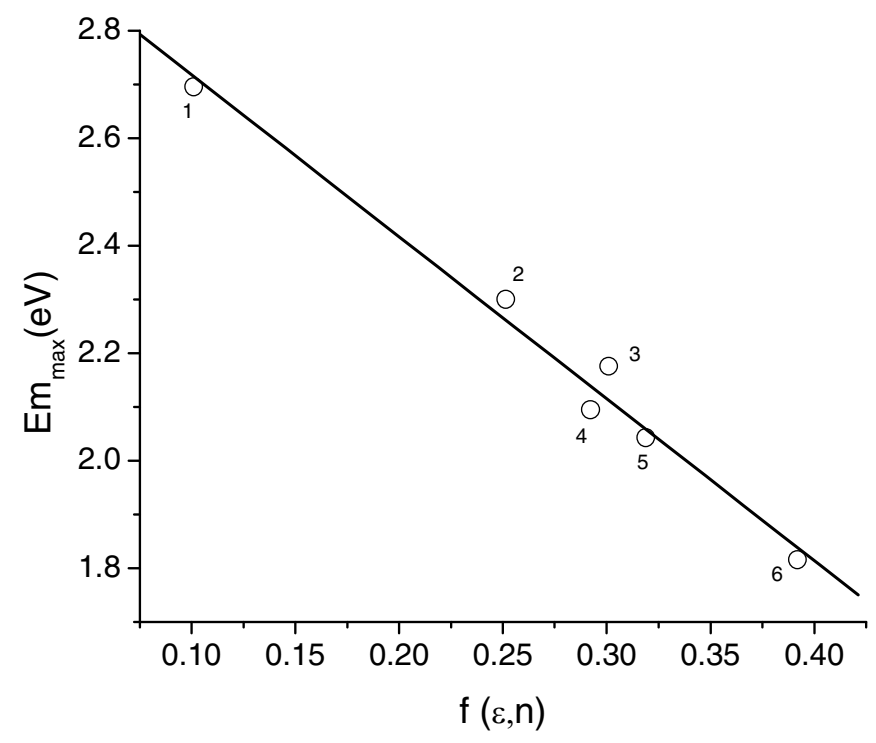

Figure S2. Lippert-Mataga plot for DMJ-An $\left(\mathrm{R}^{2}=0.981\right.$ where $\mathrm{f}(\varepsilon, \mathrm{n})=[(\varepsilon-$ $\left.1) /(2 \varepsilon+1)-0.5 \mathrm{x}\left(\mathrm{n}^{2}-1\right) /\left(2 \mathrm{n}^{2}+1\right)\right]$. The solvents are (1) methylcyclohexane, $(2)$ diethyl ether, (3) 2-methyltetrahydrofuran, (4) ethyl acetate, (5) methylene chloride, and (6) acetonitrile.

\section{Electron Transfer Reaction Energetics}

The distances between the donor, bridge, and acceptor components were determined from the energy minimized structures of DMJ-An-Ph ${ }_{\mathrm{n}}$-NI determined using semi-empirical RHF-PM3 and UHF-PM3 electronic structure calculations. ${ }^{6}$ These data are given in Table S1. Direct excitation of DMJ-An produces $\mathrm{DMJ}^{+\bullet}-\mathrm{An}^{-\bullet}$, which has a measured energy of $E_{\mathrm{CT}}=2.89 \mathrm{eV}$ in toluene, so that the energies of the ion pairs produced by subsequent electron transfers can be calculated from the corresponding changes in redox potentials and the change in Coulomb energies as the distances between the ion pairs change: ${ }^{1}$

$$
\begin{aligned}
\Delta G_{F}=\Delta G_{I}+\operatorname{sign}\left(E_{I}-E_{F}\right)+\frac{e^{2}}{\varepsilon_{S}}\left(\frac{1}{r_{I}}-\frac{1}{r_{F}}\right) & \text { if }\left|E_{F}\right|>\left|E_{I}\right|, \text { then } \operatorname{sign}=(-) ; \\
& \text { if }\left|E_{I}\right|>\left|E_{F}\right|, \text { then } \operatorname{sign}=(+)
\end{aligned}
$$

where $\Delta G_{\mathrm{I}}$ and $\Delta G_{\mathrm{F}}$ are the energies above ground state for the initial and final ion pairs, 
respectively, $E_{\mathrm{I}}$ and $E_{\mathrm{F}}$ are the redox potentials for the initial and final ions, respectively, between which the electron is transferred, $r_{\mathrm{I}}$ and $r_{\mathrm{F}}$ are the initial and final ion pair distances, respectively, $e$ is the electronic charge, and $\varepsilon_{\mathrm{S}}$ is the static dielectric constant of the solvent $\left(\varepsilon_{\mathrm{S}}=\right.$ 2.38 for toluene ${ }^{8}$ ). The one-electron oxidation potentials for $\mathrm{DMJ}, \mathrm{Ph}, \mathrm{Ph}_{2}, \mathrm{Ph}_{3}$, and $\mathrm{Ph}_{4}$ are 0.63 , $2.4,1.85,1.60$, and $1.47 \mathrm{~V}$ vs. SCE, respectively. ${ }^{9}$ The one-electron reduction potentials for $\mathrm{NI},{ }^{10} \mathrm{Ph}, \mathrm{Ph}_{2}, \mathrm{Ph}_{3}$, and $\mathrm{Ph}_{4}$ are $-0.53,-3.35,-2.68,-2.40$, and $-2.28 \mathrm{~V}$ vs. SCE, respectively. ${ }^{9}$ The results of these ion pair energy calculations are given in Table S1.

Table S1. Ion pair distances and energies of ion pair states

\begin{tabular}{|l|l|l|l|l|l|l|l|}
\hline & $\begin{array}{l}r_{\text {An-NI }} \\
(\AA)\end{array}$ & $\begin{array}{l}r_{\text {DMJ-NI }} \\
(\AA)\end{array}$ & $\begin{array}{l}r_{\text {DMJ-Ph }} \\
(\AA)\end{array}$ & $\begin{array}{l}r_{\mathrm{Ph}-\mathrm{NI}} \\
(\AA)\end{array}$ & $\begin{array}{l}-\Delta G_{\mathrm{DMJ}+\mathrm{NI}-} \\
(\mathrm{eV})\end{array}$ & $-\Delta G_{\mathrm{DMJ}+\mathrm{Ph}-}$ & $-\Delta G_{\mathrm{Ph}+\mathrm{NI}-}$ \\
$(\mathrm{eV})$ & $(\mathrm{eV})$ \\
\hline $\mathbf{1}$ & 10.4 & 15.8 & 9.6 & 6.2 & 2.16 & 4.73 & 3.34 \\
\hline $\mathbf{2}$ & 14.7 & 20.1 & 11.8 & 8.3 & 2.24 & 4.18 & 3.03 \\
\hline $\mathbf{3}$ & 19.0 & 24.4 & 13.8 & 10.6 & 2.29 & 3.97 & 2.94 \\
\hline $\mathbf{4}$ & 23.4 & 28.8 & 16.1 & 12.7 & 2.33 & 3.92 & 2.90 \\
\hline
\end{tabular}

\section{Distance Dependence of Solvent Reorganization Energy}

The distance dependence of the solvent reorganization energy $\lambda_{\mathrm{S}}$ was calculated using the Marcus expression, ${ }^{11}$ which is based on the dielectric continuum model of the solvent:

$$
\lambda_{S}=e^{2}\left(\frac{1}{2 r_{D}}+\frac{1}{2 r_{A}}-\frac{1}{r_{D A}}\right)\left(\frac{1}{\varepsilon_{0}}-\frac{1}{\varepsilon_{S}}\right)
$$

where $e$ is the electronic charge, $r_{\mathrm{D}}$ and $r_{\mathrm{A}}$ are the radii of the donor and acceptor, respectively, $\varepsilon_{\mathrm{S}}$ is the static dielectric constant, and $\varepsilon_{0}$ is the optical (high frequency) dielectric constant, which is 
approximated as the index of refraction squared. The radii of $\mathrm{An}^{-\bullet}, \mathrm{DMJ}^{+\bullet}$, and $\mathrm{NI}^{-}$are all approximately $4 \AA$, and the distance for electron transfer from $\mathrm{An}^{-\bullet}$ to $\mathrm{NI}^{*}$ spans a range of 10.4 $\AA$ for 1 to $23.4 \AA$ for $\mathbf{4}$, Table S1, so that using equation $2, \varepsilon_{S}=2.38$ and $\varepsilon_{S}=2.25$ for toluene, $\lambda_{S}$ $=0.054 \mathrm{eV}$ and $0.073 \mathrm{eV}$, respectively. For charge recombination the distance between $\mathrm{DMJ}^{+\bullet}$ and $\mathrm{NI}^{\bullet}$ ranges from $15.8 \AA$ for $\mathbf{1}$ to $28.8 \AA$ for $\mathbf{4}$, Table $\mathrm{S} 1$, so that using equation $2, \varepsilon_{\mathrm{S}}=2.38$ and $\varepsilon_{\mathrm{S}}=2.25$ for toluene, $\lambda_{\mathrm{S}}=0.065 \mathrm{eV}$ and $0.075 \mathrm{eV}$, respectively. Thus, the maximum change in $\lambda_{\mathrm{S}}$ over these distances is $<0.02 \mathrm{eV}$, so that the distance dependencies of the rates of these reactions should depend only weakly on $\lambda_{\mathrm{s}}{ }^{11}$ 


\section{References:}

(1)Greenfield, S. R.; Svec, W. A.; Gosztola, D.; Wasielewski, M. R. J. Am. Chem. Soc. 1996, $118,6767-6777$.

(2)Weiss, E. A.; Ahrens, M. J.; Sinks, L. E.; Gusev, A. V.; Ratner, M. A.; Wasielewski, M. R. J. Am. Chem. Soc. 2004, 126, 5577-5584.

(3)De Barry Barnett, E.; Cook, J. W.; Matthews, M. A. Recueil des Travaux Chimiques des Pays-Bas et de la Belgique 1925, 44, 217-223.

(4)Lippert, E. Z. Naturforsch 1955, 10a, 541-546.

(5)Mataga, N.; Kaifu, Y.; Koizumi, M. Bull. Chem. Soc. Jpn. 1956, 29, 465-470.

(6)Hyperchem(TM) Hypercube Inc. 1115 NW 4th Street, G., Florida 32601, USA.

(7)Herbich, J.; Kapturkiewicz, A. Chem. Phys. 1991, 158, 143-153.

(8)Lide, D. R.; Kehiaian, H. V. CRC handbook of thermophysical and thermochemical data; CRC Press: Boca Raton, 1994.

(9)Meerholz, K.; Heinze, J. Electrochim. Acta 1996, 41, 1839-1854.

(10)Gosztola, D.; Niemczyk, M. P.; Svec, W. A.; Lukas, A. S.; Wasielewski, M. R. J. Phys. Chem. A 2000, 104, 6545-6551.

(11)Marcus, R. A. J. Chem. Phys. 1956, 24, 966-978. 